\title{
ASTRONOMY IN UKRAINE
}

\author{
Status of Astronomical Research
}

\author{
YA.V.PAVLENKO \\ Main Astronomical Observatory of the National Academy of \\ Sciences, 27 Zabolotnoho, Kyiv-127, 03680 Ukraine \\ Centre for Astrophysics Research, University of Hertfordshire, \\ College Lane, Hatfield, Hertfordshiere AL10 9AB, UK \\ I.B.VAVILOVA \\ Dobrov Center for Scientific-Technical Potential and History \\ of Science Research, National Academy of Sciences of Ukraine \\ 60 T.Shevchenko Blvd., Kyiv 01032 Ukraine \\ AND \\ T. KOSTIUK \\ NASA/Goddard Space Flight Center, Greenbelt, MD 20771, \\ $U S A$
}

\begin{abstract}
The current and prospective status of astronomical research in Ukraine is discussed. A brief history of astronomical research in Ukraine is presented and the system organizing scientific activity is described, including astronomy education, institutions and staff, awarding higher degrees/titles, government involvement, budgetary investments and international cooperation. Individuals contributing significantly to the field of astronomy and their accomplishments are mentioned. Major astronomical facilities, their capabilities, and their instrumentation are described. In terms of the number of institutions and personnel engaged in astronomy, and of past accomplishments, Ukraine ranks among major nations of Europe. Current difficulties associated with political, economic and technological changes are addressed and goals for future research activities presented.
\end{abstract}

\section{Introduction}

Since resuming its independence in 1991 Ukraine has been striving towards social democracy and a market-based economy. In 2005 after the "Orange 
Revolution" these goals as well as European integration became priorities of Ukraine's policy. Science, and in particular astronomy, also has to adopt a model that matches the emerging market-based economy. Recently Ukrainian science has experienced complicated institutional and structural changes in the state-administered system formed during Soviet times. The basics principles of the reforms are: to match scientific endeavors to the economic capabilities of the nation; to form a mechanisn to address legal and economic issues, including protection of intellectual rights and appropriate coordinatation and budgeting for effective governing of the scientific and technological field; to specify science and engineering development priorities; to radically improve the resource management and to address the effects of aging and the shortage of personnel; to follow the principle of "openness" in science; and to promote wide international cooperation (Yatskiv \& Vavilova 2003).

\subsection{GEOGRAPHICAL LOCATION}

Ukraine is one of the largest countries on the European continent covering $603,700 \mathrm{sq} . \mathrm{km}$ in area. Its territory stretches $1316 \mathrm{~km}$ east-west and $893 \mathrm{~km}$ north-south. Ukraine borders Poland, Slovakia, Hungary, and Romania in the west, Moldova in the southwest, Belarus in the north, and Russia in the northeast and east (Fig. 1). The southern frontiers of Ukraine are washed by the Black Sea and the Sea of Azov with a coastline equal to $2835 \mathrm{~km}$.

\subsection{POPULATION}

Ukraine's population on October 1, 2004 was 47383486

(www.ukrcensus.gov.ua/results). The population is ethnically diverse with $77.8 \%$ ethnic Ukrainian. The urban population is about $67.2 \%$ and the population density is about 80 inhabitants per sq. $\mathrm{km}$.

\subsection{POLITICAL SYSTEM AND GOVERNMENT}

Ukraine has a presidential political system with a one house parliament (Verkhovna Rada). Some changes strengthening the parliament were introduced during the "Orange Revolution" but are scheduled to come into effect in 2006.

The capital is Kyiv (Kiev) with 2611000 inhabitants. The state administrative system consists of the Autonomic Republic of Crimea with Simferopol its capital, 24 regions (i.e., oblasts), 481 administrative-territorial districts. The cities of Kyiv and Sevastopol (Crimea) have a special administrative status. There are 451 cities, 893 towns, and 28651 villages in the country. 


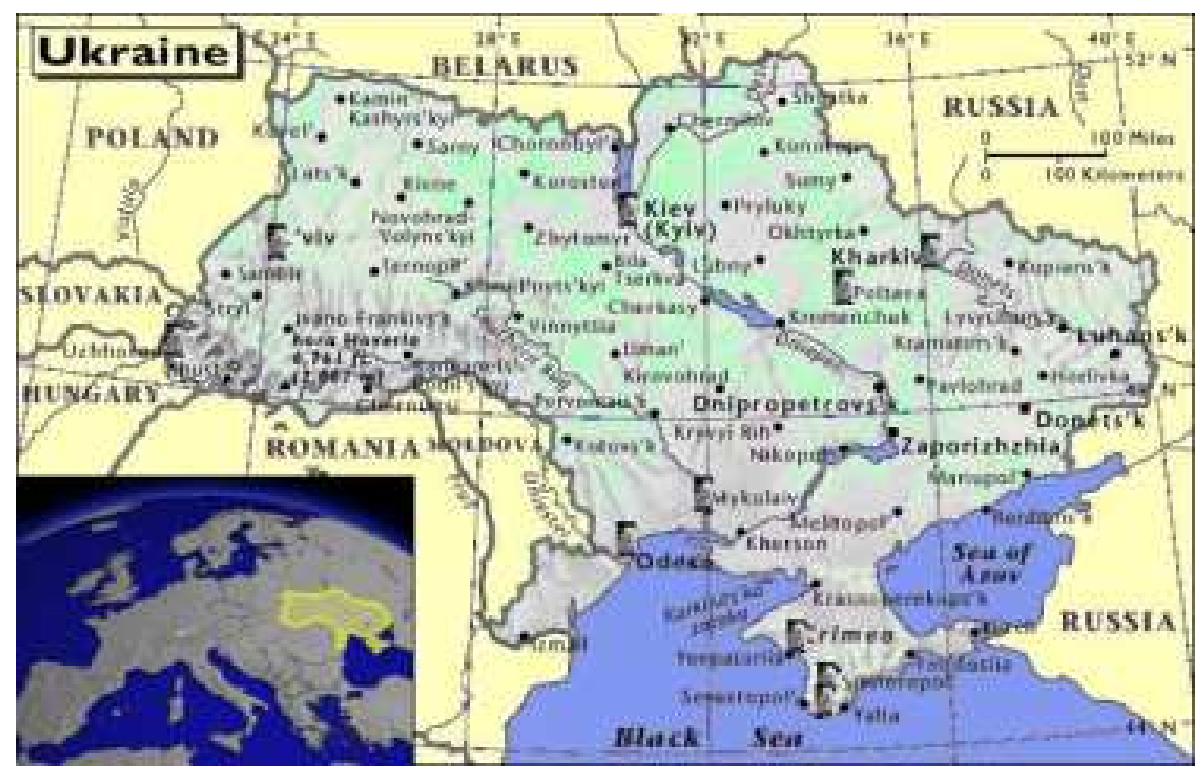

Figure 1. Astronomical sites in Ukraine

\subsection{EDUCATION}

Ukraine is a highly-educated country where 28900000 citizens have received a secondary or higher education. In the age group of 18 years and older, 13700000 persons have some form of higher education

(www.ukrcensus.gov.ua).). It also is important to note that the full legal age in Ukraine is 18 years and coincides with the age when a citizen completes his/her secondary education.

At present, there are 48,000 educational institutions, where 10 million scholars and students are being trained by 1.5 million teachers and lecturers. Among them, 2700000 citizens (of which 54\% are females) are students of 966 higher educational institutions. There, 178000 lecturers are engaged in the educational process - 44500 have a Candidate of Science degree, 7400 have a Doctors of Science degree, among them 7500 have the academic status of Professor and 31600 of Assistant Professor (Nikolaenko 2004). Two hundred higher educational institutions have University status or National University status. At present, the Ministry of Education and Science of Ukraine, MESU, which is responsible for development of education, is working to improve the efficiency of this system in the framework of the Bologna Process (http://www.aic.lv/ace/ace_disk/Bologna/index.htm 
or http://www.pfmb.uni-mb.si/bologna/Joint\%20declaration\%20of\%20the \%20European\%20Ministers\%20of.htm). This Process aims to standardize the various European higher education systems with the objective of creating a European Area of Higher Education and of promoting the European higher education system worldwide. On May 19, 2005 Ukraine joined the Bologna Process.

\subsection{SCIENCE}

There are three governmental structures which are mostly responsible for development of science and technology (S\&T): the National Academy of Sciences of Ukraine (NASU), the Ministry of Industrial Policy, and the Ministry for Education and Science. Beginning in 1991, the total expenditure (budgetary and off-budgetary) for research and development (R\&D) has been reduced by factor of 4 (750 000000 USD in 2004). The gross expenditure on R\&D as a percentage of GDP also has been reduced by a factor of two. The total budgetary expenditure on S\&T in $1991-2002$ in relative terms of purchasing-power parity has also deteriorated by one-half to 2030000 USD in 2002 (Yatskiv 2004).

Science in Ukraine, including astronomical research, is now facing a difficult time due to economic limitations of the nation and the need for upgrading the existing scientific infrastructure. The key problems are both the low GDP activities and the fact that even the low budgetary expenditures on science and technology have not been effectively spent. In 2005 the adopted budget is 20400000000 USD with 5\% devoted to R\&D.

In 2004 about 173000 employees were engaged in science and technology activity: $54 \%$ of them worked in industry, $28 \%$ in national academies of science, and $18 \%$ in higher educational institutions. In this group 73700 persons are Candidates and Doctors of Science As was mentioned above, $70 \%$ of them work in higher educational institutions. If one considers the number of researchers per 10000 labor force, we see that Ukraine with 41 is at the level of countries such as, Germany - 58, Great Britain - 54, Austria -34 .

Beginning in 1991, there has been an increase in the number of postgraduate students (13 600 in 1991 and 24500 in 2003). Although the total number of scientists has decreased during this period because of a "brain drain" both outside and inside Ukraine, the number of Doctors and Candidates of Sciences has been slowly increasing. A greater fraction of those taking science and engineering degrees during this period were women: $54 \%$ of graduate students, $54 \%$ of Candidates of Science, $27 \%$ of Doctors of Science, and $10 \%$ of members of academies of sciences. 


\section{Brief History of Astronomical Research}

Astronomical culture and research have long-standing traditions in the country. The first signs of astronomical knowledge were found in archaeological excavations and records. The most ancient find (dated as 15000 B.C.) is a mammoth tusk with a fretwork image of a table of lunar phases found in the Poltava region. The so-called Trypillya culture (dated $4000-3000$ B.C) had numerous examples of ornaments at the howls, distaffs, wheels and other everyday articles with symbolic images of zodiac constellations, and vessel-calendars indicating the vernal/autumnal equinoxes and the motion of the Sun.

Another unique historical record relates to the times of the powerful state of the Kievan Rus' (X - XIII centuries), when astronomical observations were conducted mainly in cloisters. For example, the authors of the Lavrentievska chronicle describe the solar eclipses of the years 1064, 1091, and 1115 A.D. and the lunar eclipses of 1161 A.D.

The first book on astronomy written in the modern territory of Ukraine was by physician and astronomer Georgii Drohobich in 1483, who later became the Rector of Bologna University (http://litopys.org.ua/human /hum47.htm). A graduate of the Kyiv-Mohyla Academy, Ivan Kopievsky, issued the first stellar map in the Slavic language in Amsterdam in 1699 and the basics of naval astronomy in 1701. The prominent UkrainianRussian philosopher, scientist and religious figure, Pheophan Prokopovich, who worked at the Kyiv-Mohyla Academy in 1705 - 1716 and was rector in 1711 - 1716, lectured astronomical courses based on theories of Copernicus and Galileo. He also developed the philosophical foundations of the unity of matter and motion, which were generalized later by Mikhail Lomonosov.

The first astronomical observatory on the territory of western Ukraine was founded in L'viv University in 1769. The first astronomical observatory in Kyiv was founded in the library of the Kyiv-Mohyla Academy in 1783 and was equipped with modern instruments of those times. In 1821 the Naval Observatory was established in Mykolaiv, which functioned in 1912-1991 as a department of the Pulkovo Observatory (St. Petersburg, Russia). Later on university observatories were founded in Kyiv (1845), Odesa (1871), and Kharkiv (1883). In 1909 a southern department of the Pulkovo observatory was established in Simeiz (Crimea). Being engaged in the educational process, these observatories conducted research in astrometry, theoretical astronomy, and astrophotometry.

The Ukrainian Academy of Sciences was founded in 1918. This resulted in a new impetus for the development of science and technology, in particu-

lar, the establishment of new astronomical institutions and infrastructure. Among them are the Gravimetric Observatory in Poltava (1926), which 
is now a division of the Institute of Geophysics; the Main Astronomical Observatory, MAO, in Kyiv (1944); the Crimean Astrophysical Observatory, CrAO, in Naukove, Crimea (1945); the Radio Astronomical Observatory of the Institute of Radio Physics and Electronics in Grakovo (1958), which later became a division of the Institute of Radio Astronomy, IRA, in Kharkiv (1985); the High-Altitude Observatory at the Peak Terskol, the North Caucasus (1970's) which later became a division of the International Center for Astronomical and Medical-Ecological Research, ICAMER (1992). Currently, the astronomy sector of the National Academy of Sciences of Ukraine is well-developed as compared to that at the universities.

The turning points in the history of science are inseparably linked with outstanding personalities. Among them are the founders of modern astronomy in Ukraine who obtained world recognized achievements in the years 1920 - 1970: A. Y. Orlov and E.P.Fedorov (astrometry, complex research of the Earth rotation); N. P. Barabashov (photometry of planets, the first catalogue of details of the Lunar surface, developer of one of the first spectrohelioscopes for research of the solar photosphere and chromosphere); G.A.Shajn (stellar evolution, kinematics and the magnetic field of the Galaxy, solar corona, long-periodic variable stars, discovery and research of new emission nebulae); A.B.Severnyj (spectral research of solar flares and other non-stationary processes on the Sun, magnetic fields of the Sun and stars, building of one of the largest solar telescopes, the Tower Solar Telescope, solar oscillations, helioseismology, the problem of solar deuterium, development of space-born telescopes and research programs for space missions); V.P. Tsesevich (complex research of variable stars); A.Y.Yakovkin (research of the Moon); S.K.Vsehsvyatskij (cometary research, theories on volcanic activity on satellites and the existence of rings around Jupiter, independent discovery of the solar wind altogether with Ponomariov); S.A.Kaplan (general relativity theory, white dwarfs, gasdynamical processes); S.Ya.Braude, one of the founders of decameter radio astronomy (developer/builder of the largest decameter telescope UTR-2 and the decameter VLBI URAN system, author of the first catalogue of extragalactic objects in the decameter radio range).

\section{The Current Status of Astronomical Research}

\subsection{SECONDARY AND HIGHER ASTRONOMICAL EDUCATION}

\subsubsection{Secondary Education}

Without exaggeration we can say that the system of education and its achievements at this level were the most extraordinary accomplishments of the former Soviet Union, in particular as it concerned education in astronomy. Astronomy was a basic course in secondary schools (34 academic 
hours in the last, 10th, grade). Nobody knows the reasons, although it is possible it was an echo of reform, but after Ukraine resumed its independence in 1991, from 1992 until 2000 astronomy was excluded from the secondary education basic curriculum. In 2000, as a result of persistent activity by the Ukrainian Astronomical Association (UAA) and numerous round-tables with representatives of ministry departments, this regretable decision was corrected and astronomy was reinstated into the current 12year secondary education curriculum.

The present-day status of the astronomical education in secondary schools is as follows (Vavilova \& Yatskiv 2003):

Some elements of the astronomical discipline are included in the standard "Natural Science" curricullum of the 6th - 11th grades.

"Astronomy" is a required course in general (non-specialized) schools (17 academic hours in the last 12th grade) and in lyceums of the natural sciences (34 academic hours in the 12th grade).

"Astronomy" as an ellective course is studied in gymnasiums of the humanities.

By comparing the status of secondary astronomical education in other countries (e.g., Russia, several countries of Europe) the UAA timely improved this situation in Ukraine by arguing that knowledge of astronomy will play a unique role for generations to come in the twenty-first century. Our present-day problems in this improvement are how to increase the number of new textbooks and how to organize regular training of astronomy teachers.

Several planetariums are open to the public. Two of them, in Kyiv and Kharkiv, are located in separate buildings. The Drahomanov Pedagogical University of Kyiv, the Pedagogical University of Mykolaiv, the National University of Uzhgorod also train teachers of astronomy for secondary schools.

\subsubsection{Higher astronomical education}

We list below the most important national universities, which have astronomy and space related faculties:

- Shevchenko National University of Kyiv (www.univ.kiev.ua)

- V.N. Karazin National University of Kharkiv (www.univer.kharkov.ua)

- I.I. Mechnikov National University of Odesa (www.onu.edu.ua)

- Ivan Franko National University of L'viv (www.franko.lviv.ua)

- National Technical University "Kyiv Polytechnical Institute" (www.ntu-kpi.kiev.ua )

- National University of Dnipropetrovsk (www.dsu.dp.ua)

- V.I. Vernadsky Taurian National University in Simferopol (www.ccssu.crimea.ua/tnu) 
- National University of Uzhgorod (www.univ.uzhgorod.ua)

- Zhukovsky National Aerospace University in Kharkiv (www.xai.edu.ua)

All astronomical programs are structural divisions of the physics departments of universities. For this reason as well as the fact that the Ukrainian system of university education in the natural sciences is similar to that of the German system, our students-astronomers receive good training in mathematics and physics.

Every year a total of about 75 university entrants are educated in astronomy. After the 4th year they obtain a Bachelor diploma in Physics and on graduating from the university they obtain either a Diploma of Specialist or a Master's Degree in Astrophysics/Astronomy. They study the classical university courses (astrometry, celestial mechanics, planetary physics, solar physics, astrophysics, applied astrophysics, theoretical astrophysics, extragalactic astronomy etc.) as well as special courses on contemporary astronomical research, and have seminars and training in observational astronomy. Results of our monitoring show that $80 \%$ of the entering students finish their education in 5 years; $50 \%$ of students, who finished their education, continue to work in astronomy; $30 \%$ of holders of a Specialist's diploma or Master's Degree defend a Candidate Thesis within 3 - 7 years after they graduate (Vavilova \& Yatskiv 2003).

Current problems in implementation of the astronomical education program are the following:

- There are no required astronomical courses in the first to third year of education in the bachelor programs (only classical courses for physicists)

- Many textbooks need to be renewed

- There is a drop of the mean level in the ability of our students during the last few years and an indication of this problem is also evident in secondary education

- The need to raise the prestige of the scientific profession irrespective of the low salaries for young scientists and engineers.

\subsection{SCIENTIFIC DEGREES AND SCIENTIFIC CAREERS OF ASTRONOMERS}

\subsubsection{Scientific degrees}

The system of Bachelor and Master's degrees was initiated in 2002. For this reason we are not ready yet to analyze how it works. The system of higher scientific degrees in Ukraine is inherited from the Soviet type system and consists of two levels: Candidate of Science, and Doctor of Science. During recent years the Ministry of Foreign Affairs of Ukraine approved memorandums about compliance of diplomas of Candidate of Science and 
$\mathrm{PhD}$ with dergrees of more then 100 countries of the world. Everybody with a degree up to the Cand. Sci. Diploma may obtain a certificate of compliance for work abroad.

The first level degree of Candidate of Sciences (Cand. Sci.) is the analogue of the degree of Doctor of Philosophy or Doctor of Medicine adopted in Europe and other countries. The Cand. Sci. degrees unlike the Ph.D. degrees are classified by the related scientific fields (chemistry, biology, pedagogy, economy, politics etc.). The second level degree of Doctor of Science (Dr. Sci.) is also classified by related scientific fields. The Degrees of Cand.Sci. and Dr.Sci. for those who work in astronomy are related to such fields as the Physics-Mathematical Sciences and Technical Sciences.

As a rule, the Cand. Sci. thesis in astronomy is a manuscript (90-120 pages) based on at least 3 papers published in refereed journals and resulting in a new achievement in astronomy. The Dr.Sci. thesis is a manuscript (220 - 260 pages) based on: a) significant scientific discovery in Astronomy; b) advanced results published in at least 20 papers in refereed journals; c) an author's monograph.

The thesis of Cand. Sci. and Dr. Sci. is defended following a special procedure. The first step is a report at a Scientific Council meeting of the institution where the thesis has been conducted. This Scientific Council recommends/does not recommend a thesis defense. The second step is a report at the meeting of the Special Council on Thesis Defense, which nominates/does not nominate the candidate for a Cand.Sci. or Dr.Sci. degree as well as decides whether the thesis satisfies the requirements of a Cand. Sci. or Dr. Sci. thesis. Candidates for a Cand. Sci. degree must also take exams in philosophy, foreign language, and in their specialty before the defense.

For defense of the Cand. Sci. thesis the Special Council selects two reviewers, 2 Drs.Sci, or 1 Dr.Sci and 1 Cand. Sci., who will study the manuscript in details and prepare reports. For defense of Dr. Sci. thesis, the Special Council selects three reviewers (3 Dr.Sci.) with a backgroud in a field relevant to the defended thesis. In both cases one astronomical institution in the relevant scientific field is appointed to be an independent "Leading Referee Institution". The signed reviews must be approved by the Director of this institution.

In the third step, the Supreme Attestation Commission of Ukraine approves/does not approve this thesis as well as awards/does not award the Cand.Sci. and Dr.Sci. degree. The Supreme Attestation Commission (SAC) of Ukraine (www.vak.org.ua) is a government institution, which develops the general rules for processing thesis manuscripts, thesis defense and approval as well as awards the scientific degrees. This institution also approves the membership of the Special Councils on Thesis Defense. The Special 
Expert Councils under SAC were established to consider possible conflict situations. Members of these special councils must have a Dr. Sci. degree in a specialty relevant to that of the defended thesis. Membership in these councils is renewed once every three years.

In the current system a positive defense at the Special Council meeting is possible even when a negative report is prepared by the reviewers. The Special Council may also decide that the Cand. Sci. thesis satisfies requirements for the Dr. Sci. thesis and can nominate the candidate for the higher Dr. Sci. Degree. In case of a conflict situation the SAC Expert Council has the right to reverse the decision of the Special Council on Thesis Defense following established procedures. It also is possibile to prepare and to defend a thesis in an interdisciplinary field, e.g., astronomical instrumentation, which is more related to the SAC requirements for theses in Technical Sciences, history of astronomy or methodology of astronomy education.

At present, the Special Council on Thesis Defense which operates at the Main Astronomical Observatory of the NASU in Kyiv, nominates astronomers for Dr. Sci. and Cand. Sci. in Phys.\& Math. It covers all the SAC adopted specialties for astronomers: "Heliophysics and Physics of Solar System", "Astrophysics and Radio Astronomy", "Astrometry and Celestial Mechanics", and "Methods of Remote Sensing of the Earth". The Special Council operated in I.I. Mechnikov National University of Odesa nominates for Cand. Sci. degree in the fields of: "Theoretical physics", "Astrophysics and Radio Astromomy". The Special Council of the V.N.Karazin National University of Kharkiv nominates for Dr. Sci. and Cand. Sci. degrees in "Astrophysics and Radio Astromomy".

Since 1992, about 130 astronomers were awarded higher scientific degrees (20\% of them Dr. Sci. Degree). Our present-day problem is a brain drain of young scientists: currently $50 \%$ of those who obtained a Cand. Sci. degree work outside of Ukraine.

\subsubsection{Scientific Careers.}

The nomenclature of scientific positions is the following:

Junior Staff Scientist - holders of a Master's degree who are starting the scientific activity.

Research Staff Scientist- scientists who defended Cand. Sci. thesis.

Senior Staff Scientist - those who worked successfully for at least five years as Research Staff Scientist.

Leading Staff Scientist - scientists who defended Dr. Sci. Thesis.

Principal Staff Scientist - senior scientist who worked successfully at the position of Leading Staff Scientist.

The nomenclature of faculty positions in higher educational institutions 
is the same as in many countries: Lecturer, Senior Lecturer, AssistantProfessor, Professor.

Unfortunately, for the time being, the Soviet type system of long-term fixed staff positions has been preserved without substantial changes. There is little competition for permanent positions. In fact, any young scientist can get a position at an astronomical institution practically for the rest of his or her life. Post doctoral positions are not part of the current structure of scientific positions.

The following academic titles/status are used for scientists:

Senior Researcher - those who defended Cand. Sci. thesis and worked successfully at least two years at the position of Senior Staff Scientist

Assistant-Professor - those who defended Cand. Sci. thesis and worked at least three years at the position of Assistant-Professor

Professor - Assistant-Professors who defended the Dr. Sci. Thesis, and Senior Researchers who defended the Dr. Sci. thesis and were supervisors of at least five Candidates of Science

Corresponding Member of the National Academy of Sciences of Ukrainesenior scientists

Member of the National Academy of Sciences of Ukraine - advanced senior scientists.

\subsection{GOVERNMENT AND NON-GOVERNMENT ASTRONOMICAL INSTITUTIONS}

\subsubsection{Organization of Scientific Activity in Astronomical Institutions}

All questions and decisions concerning scientific activities (research programs, scientific careers of employees, nominations for academic status, final reports for various projects, scientific theses research, structure and staff of departments and laboratories etc.) must be considered and approved by the Scientific Council of an institution. The Council is formed by the Director of the institution and consists of the senior, leading and principle staff scientists. Membership in this council must be approved by a majority vote of scientists of the institution. Each member of the Council has one vote. A majority vote is sufficient to approve most decisions, however, nominations for staff positions and scientific titles still require a $2 / 3$ majority of total members.

Research programs can be divided into two types, depending on the sources of funding: government budget programs and off-budget programs (see sections 3.6 and 4 for details). The Director, heads of departments, and program managers form teams of scientists and engineers to conduct the research programs. Structure of these teams may not follow a formal distribution of staff by departments or laboratories. Still, the project manager, pricipal investigator (PI) and the main department responsible for 
the project are always known. As a rule government funded programs are planned for 3 - 5 years. After completing the project the team members write a final report, which must be refereed by another institution. The final report must then be presented and approved at a Scientific Council meeting. All decisions of the institution's Scientific Council must be approved by the Scientific Council or Scientific Bureau of a higher governing body.

The importance of the role of Director, Scientific Council, heads of departments and project managers for scientific activity of the institution depends on many subjective and objective factors and may vary from institution to institution.

Unfortunately, under current research bugetary conditions the principle "what is possible" often prevails over "what is more interesting". The heads of departments and research project managers are only the formal owners of the project's money. Due to practical reasons, to minimize possible problems and loses, usually one person (as a rule, the Director of the institution) is responsible for resolving all financial issues.

\subsubsection{Governmental Astronomical Institutions}

In general, 12 governmental institutions are engaged in astronomical scientific activity. They are governed by two different higher government level organizations: the National Academy of Sciences of Ukraine (NASU) and the Ministry of Education and Science of Ukraine (MESU).

As mentioned in Section 2, the largest astronomical institutions are established within the structure of the NASU (www.nas.gov.ua). NASU is a self-governing organization whose activity is aimed at S\&T, socialeconomical, and cultural development of Ukraine. The Government of Ukraine (www.kmu.gov.ua) forms the NASU budget. In its own turn, the NASU Presidium, through relevant scientific NASU Divisions, forms a budget of all subordinated institutions. The role of the NASU in the national basic and applied S\&T policy is the same as the Max Plank Geselschaft in Germany or the Centre National de Recherche Scientifique in France.

The following four astronomical institutions are subordinated to the NASU:

- Main Astronomical Observatory (www.mao.kiev.ua ) in Kyiv

- Institute of Radio Astronomy (www.ira.kharkov.ua) in Kharkiv

- Poltava Gravimetric Observatory (pgo@poltava.ukrtel.net ) of the Institute of Geophysics

- International Center for Astronomical \& Medical-Ecological Research (ICAMER), in Terskol, North Caucasus, Russia (www.mao.kiev.ua/icamer or (www.allthesky.com/observatories/terskol.html).

The first two institutions are related to the NASU Division for Physics 
and Astronomy, the third one to the NASU Division for Earth Sciences. The bureaus of these divisions approve decisions of Scientific Councils of the mentioned astronomical institutions. The ICAMER is an interdivisional institution and its activity is regulated in the framework of a special memorandum signed by the NASU and the Russian Academy of Sciences and by the Governments of Ukraine and Russia.

The Ministry of Education and Sciences of Ukraine (www.mon.gov.ua) governs seven institutions engaged in astronomical research. Two of them are directly subordinate to the MESU:

- Scientific Research Institute, Crimean Astrophysical Observatory, (www.crao.crimea.ua ) in Naukove, Crimea

- Research Institute "Mykolaiv Astronomical Obsevatory" (www.mao.nikolaev.ua) in Mykolaiv.

Three astronomical observatories function as Research Institutes at universities:

- Scientific Research Institute "Astronomical Observatory" of the I.I. Mechnikov National University of Odesa (astro@paco.odessa.ua)

- Institute of Astronomy of the Karazin National University of Kharkiv (www-astron.univer.kharkov.ua)

- Astronomical Observatory of the Ivan Franko National University of L'viv (www.astro.franko.lviv.ua).

Two small astronomical institutions function as departments of universities:

- Space Research Laboratory, National University of Uzhgorod (www.univ.uzhgorod.ua/nauka/zsurnal/Lab-space.html),

- N.I.Kalinenkov Astronomical Observatory, Pedagogical State University of Mykolaiv (office@mdpu.edu.ua).

These universities allocate the budgets of the observatories as well as maintain Departments of Astronomy for training students. The Scientific Councils of universities approve all decisions of the Scientific Councils of the observatories.

The Shevchenko National University of Kyiv is a self-governing institution and its budget is determined by the central government. An Astronomical Observatory functions there as a Research Laboratory of the Department of Astronomy and Space Physics of the Physics Faculty (www.observ.univ.kiev.ua).

Information on staff membership and research fields of larger astronomical institutions is presented in Table 1 (Vavilova \& Yatskiv 2003).

Taking into account quantitative factors, i.e. the number of scientists engaged in astronomical research per population, the number of astronomical institutions as well as astronomical infrastructure (see Section 3.4), we could consider Ukraine a large astronomical country in Europe. In total, 
TABLE 1. Staff membership and research fields of larger astronomical institutions in Ukraine

\begin{tabular}{|c|c|c|}
\hline Institution & $\begin{array}{l}\text { Total staff/ } \\
\text { Scientific staff/ } \\
\text { Cand and Dr. Sci. }\end{array}$ & $\begin{array}{l}\text { Recearch } \\
\text { fields }\end{array}$ \\
\hline $\begin{array}{l}\text { Main Astronomical } \\
\text { Observatory } \\
\text { of the NAS of Ukraine }\end{array}$ & $213 / 90 / 69$ & $\begin{array}{l}\text { Extragalactic Astronomy } \\
\text { Physics of stars \& brown dwarfs } \\
\text { Positional Astronomy } \\
\text { Solar System Bodies } \\
\text { Solar Physics } \\
\text { Space Geo-dynamics } \\
\text { Space Plasma Physics }\end{array}$ \\
\hline $\begin{array}{l}\text { Scientific Research Institute } \\
\text { "Crimean Astrophysical } \\
\text { Observatory" } \\
\text { of the MES of Ukraine }\end{array}$ & $358 / 92 / 58$ & $\begin{array}{l}\text { Extragalactic Astronomy } \\
\text { Ground-based and } \\
\text { Space-born Instrumentation } \\
\text { Radio Astronomy (mm, cm) } \\
\text { High-energy astrophysics } \\
\text { Physics of stars } \\
\text { Solar System Small Bodies } \\
\text { Solar Physics, Solar Activity }\end{array}$ \\
\hline $\begin{array}{l}\text { Institute of Radio Astronomy } \\
\text { of the NAS of Ukraine }\end{array}$ & $306 / 102 / 88$ & $\begin{array}{l}\text { Radio astronomy } \\
\text { (decameter and } \mathrm{mm} \text { ) }\end{array}$ \\
\hline $\begin{array}{l}\text { Astronomical Observatory of } \\
\text { the Shevchenko National } \\
\text { University of Kyiv }\end{array}$ & $64 / 35 / 26$ & $\begin{array}{l}\text { Astrometry } \\
\text { General Relativity } \\
\text { Extragalactic Astronomy } \\
\text { Solar Physics, Solar Activity } \\
\text { Solar System Small Bodies }\end{array}$ \\
\hline $\begin{array}{l}\text { Astronomical Observatory } \\
\text { of the Ivan Franko National } \\
\text { University of L'viv }\end{array}$ & $28 / 16 / 12$ & $\begin{array}{l}\text { Extragalactic Astronomy } \\
\text { Cosmology } \\
\text { Satellite Geodesy } \\
\text { Solar Physics, Solar Activity }\end{array}$ \\
\hline $\begin{array}{l}\text { Scientific Research Institute } \\
\text { "Astronomical Observatory" } \\
\text { of the I.Mechnikov } \\
\text { National University of Odesa }\end{array}$ & $75 / 65 / 26$ & $\begin{array}{l}\text { Physics of the Solar System } \\
\text { Small Bodies } \\
\text { Variable stars } \\
\text { Physics of stars }\end{array}$ \\
\hline $\begin{array}{l}\text { Institute of Astronomy of } \\
\text { V. Karazin National } \\
\text { University of Kharkiv }\end{array}$ & $83 / 43 / 20$ & $\begin{array}{l}\text { Ground-based Instrumentation } \\
\text { Physics of stars } \\
\text { Solar activity } \\
\text { Solar System Small Bodies }\end{array}$ \\
\hline $\begin{array}{l}\text { Mykolaiv Astronomical } \\
\text { Observatory } \\
\text { of the MES of Ukraine }\end{array}$ & $75 / 19 / 10$ & $\begin{array}{l}\text { Ground-based Instrumentation } \\
\text { Positional astronomy }\end{array}$ \\
\hline
\end{tabular}


more than twenty observatories and departments at various scientific institutions and universities are engaged in astronomical research. As to the qualitative factors, i.e. number of publications in world recognized journals, citation index etc., the situation is not so clear. As can be seen in Table 1, research at Ukrainian observatories covers a wide range of disciplines. In the case of observational programs, access to modern astronomical facilities is rather limited. As a result in many cases theoretical interpretations of observations conducted at other observatories still prevails. On the other hand, Ukraine has developed its own astronomical infrastructure (see section 3.4). However these astronomical facilities need to be upgraded rapidly to provide any competition for leading observatories of the world.

\subsubsection{Non-governmental Astronomical Institutions}

Since 1991 the Ukrainian Astronomical Association, UAA,

(www.observ.univ.kiev.ua/uaa) has coordinated astronomical activity in Ukraine. The UAA consists of 16 Institutional Members and dozens of Individual Members, with a total membership of about 1500 astronomers. From 1992 the UAA serves as the National Committee of the International Astronomical Union, IAU, and as an Affiliated Society of the European Astronomical Society, EAS. One hundred and sixty two Ukrainian astronomers are IAU members and 96 are EAS members.

There are a few other non-governmental institutions related to astronomy. Two of them, Odesa Astronomical Society and the Ukrainian Society of Gravitation, Relativistic Astrophysics and Cosmology, are UAA Associate Members. Two years ago the Ukrainian Society of Amateurs of Astronomy was founded under the patronage of professional astronomers.

\subsection{INFRASTRUCTURE: LARGE, MODERATE, AND SMALL TELESCOPES AND TELESCOPE NETWORKS.}

Ukrainian astronomical institutions possess a wide range of telescopes. Many of them were constructed 30 and more years ago, but are still in use. The main problem is to upgrade these telescopes and equip them with modern detectors and other devices for making observations and obtaining results of sufficient quality.

\subsubsection{Largest Telescopes and Networks, (see also Fig. 2)}

- The UTR-2, Ukrainian T-shape Radio telescope, (www.ira.kharkov.ua/ utr2), is the largest array in the world operated at the decameter wavelengths, extrememly low frequencies i25 MHz. This telescope belongs to the Institute of Radio Astronomy, IRA, of the NASU. It is located near Grakovo village, about $80 \mathrm{~km}$ from Kharkiv (northeastern Ukraine). The effective area of the UTR-2 (152 000 sq. m) is more than the ef- 

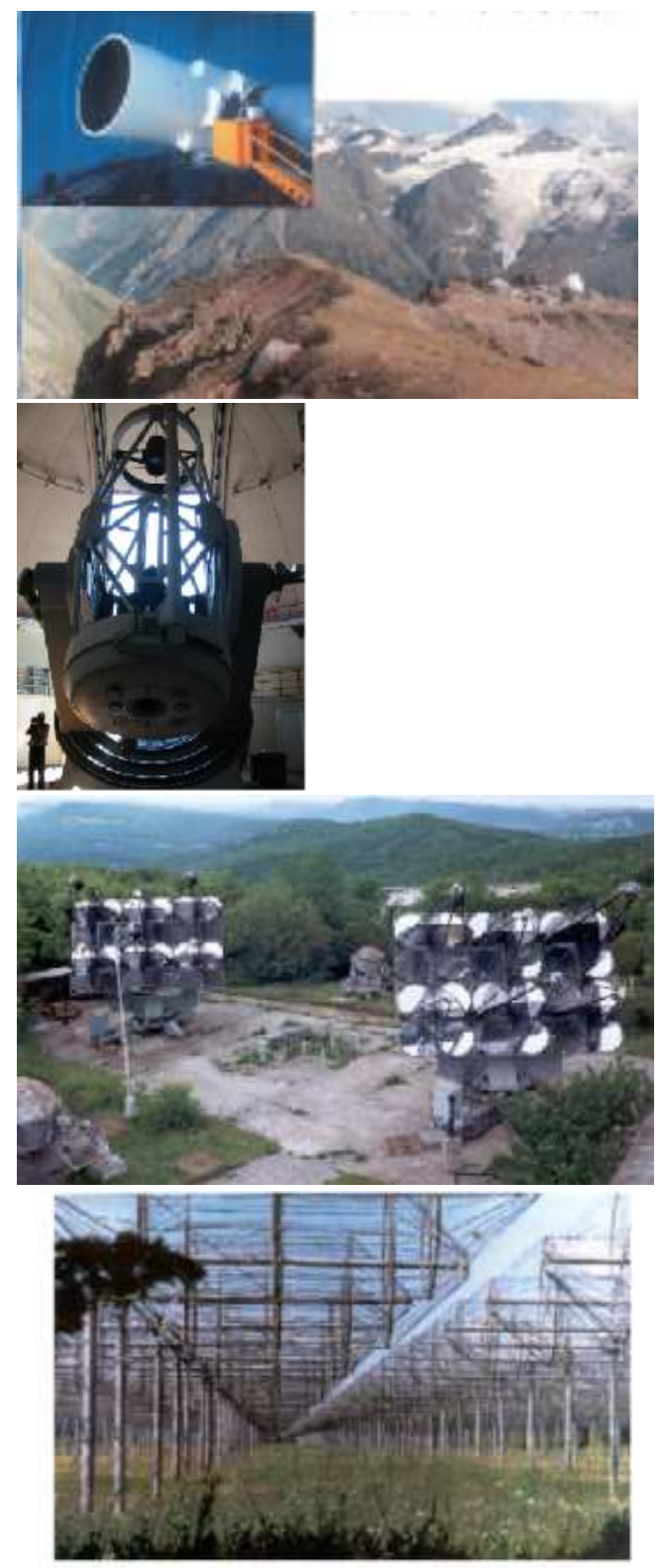

Figure 2. From top: the $2 \mathrm{~m}$ telescope of ICAMER (Peak Terskol, North Caucasus)and Acad. Shajn $2.6 \mathrm{~m}$ telescope of Crimean Astrophysical Observatory (Naukove, Crimea). Gamma Telescope GT-48 of Crimean Astrophysical Observatory and Decameter Radio Telescope UTR-2 (Kharkiv), see more on www.mao.kiev.ua/staff/yp/OSA7/a.htm. Copyright 2003 Ukrainian Astronomical Association (1,3,4) and A.V.Terebizh(2). 
fective area of all existing radio astronomical telescopes put together. The resolution is about of 40'x40' at the middle frequency, 16.7 MHz.

- A decametric Very Long Baseline Interferometry, VLBI, network URAN was built with the UTR-2 as the basis. Besides UTR-2 it consists of four additional radio telescopes with sizes 5 to 10 times less than that of UTR-2: URAN-1 near Kharkiv, URAN-2 near Poltava, URAN-3 near L'viv, and URAN-4 near Odesa. They are electrically phased steering arrays operating from 10 to $30 \mathrm{MHz}$. Baselines from $40 \mathrm{~km}$ to $900 \mathrm{~km}$ provide an angular resolution from several minutes to one second of arc. The angular resolution of 1 arcsec corresponds to the fundamental limit imposed by scattering at these frequencies in the interstellar medium. These network telescopes also belong to the Institute of Radio Astronomy.

- The 70-meter dish radio telescope RT-70 is a highly efficient fully tracking instrument located near Evpatoria in Crimea. Its effective area is of 2500 sq. $\mathrm{m}$ and its beam width is 2.5 arcmin at the 5 -cm radio wave length. There are only 10 antennas of such size in the world. This telescope is being up-graded to provide astronomical research at wavelengths $92,18.6$, and $1.35 \mathrm{~cm}$ for future work in the European VLBI network (EVN). This antenna belongs to the National Space Agency of Ukraine, NSAU.

- The RT-22 is a precise radio telescope operating at $\mathrm{mm}$ and $\mathrm{cm}$ radio wavelengths located in Simeiz, Crimea. It has a Cassegrain and prime focus feed system on an azimuth-elevation mount and its characteristics are: diameter $22 \mathrm{~m}$, surface tolerance (root mean square) $0.25 \mathrm{~mm}$, wavelength limit $2 \mathrm{~mm}$, and focal length $9.525 \mathrm{~m}$. RT-22 is included in VLBI astrophysiscal and geodetic projects with the European and USA networks. This instrument belongs to the SRI "Crimean Astrophysical Observatory" of the MESU.

- The Acadimician Shajn 2.6-meter reflector is the largest optical telescope in Ukraine. The telescope was built in 1961. Its equatorial mount supports a $2.6-\mathrm{m}$ parabolic primary with several optical systems: primary (F/4 and with a focal reducer F/2.6), Cassegrain (f/16), Nasmith (f/16), and two f/40 coude foci, direct and bent.

- 2-meter ZEISS telescope at the Peak Terskol, North Caucasus, Russia (www.allthesky.com/observatories/terskol.html). It belongs to the ICAMER.

\subsubsection{Moderate-size Telescopes}

- AZT-11 (Crimean Astrophysical Observatory) is 1.25-m Ritchey-Cretien reflector, built in 1981. Focus length is $16 \mathrm{~m}$, available foci are Main 
Cassegrain and Auxiliary Cassegrain. An offset photoelectric auto guider is provided for the Main focus. A TV guider with a 30-cm refractor and a 40 arcmin field-of-view also is available. Objects brighter then 15 mag can be resolved. A computer based control system provides automated pointing with 15 arcsec precision and other services, i.e. fine tracking of fast moving objects (comets, asteroids), access to object catalogues, and dome-telescope synchronization.

- The Tower Solar Telescope TST-1 (Crimean Astrophysical Observatory). A $120 \mathrm{~cm}$ coelostat and a $90 \mathrm{~cm}$ spherical primary mirror feed the telescope to provide $\mathrm{f} / 56$ or $\mathrm{f} / 78$ Cassegrain foci equipped with spectrographs.

- ACU-5 (Main Astronomical Observatory of the NASU, www.mao.kiev.ua/sol/sol_w1.html) consists of a 440-mm coelostat and an additional mirror, 440/17500-mm main mirror and 200-mm Cassegrain mirror system with a $60-\mathrm{m}$ equivalent focal length. The spectrograph camera and collimator mirrors are made out of one single block of glass of 500/7000-mm, the grating has a ruled area of 140x150 mm with 600 lines per $\mathrm{mm}$.

- Solar ACU-26 telescope (MAO NASU, www.mao.kiev.ua/sol/sol_w2. html) was constructed at the Peak Terskol in 1989. The diameter of the main spherical mirror is $650 \mathrm{~mm}$ with a focal length of $17750 \mathrm{~mm}$. The telescope is equipped with a 5-camera spectrograph permitting simultaneous observations in five spectral regions. The diameter of the collimator and cameras is $300 \mathrm{~mm}$, the focal length $8000 \mathrm{~mm}$. The $250 \mathrm{~mm} \times 200 \mathrm{~mm}$ grating, 600 lines/mm, permits dispersion in fourth order of $21.9 \mathrm{~mm} / \mathrm{nm}$ at $395.0 \mathrm{~nm}$ and $33.0 \mathrm{~mm} / \mathrm{nm}$ at $650.0 \mathrm{~nm}$.

- SLR (MAO, NASU, Crimean Laser Observatory in Katsiveli, Crimea) is a 100-cm telescope with Ritchey-Cretien and Coude systems on an English mounting. The equivalent focal length of Ritchey-Cretien system is $13.3 \mathrm{~m}$ and of the Coude system is $36.5 \mathrm{~m}$. A CCD-camera with $256 \times 256$ pixels allows positional and photometric observations. A satellite ranging laser is mounted at the Coude focus. The Crimean satellite laser ranging, SLR, Station N1873 is a member of a SLR world network and participates in the majority of international programs observing satellites.

- The gamma ray telescope GT-48 (Crimean Astrophysical Observatory) is designed for searching and investigating sources of very high energy (VHE) gamma radiation ( $1012 \mathrm{eV}$ ) by measuring Cherenkov flashes in the Earth atmosphere on moonless nights. The installation GT-48 consist of two independent alt-azimuth arrays $20 \mathrm{~m}$ apart. Each array consists of six 1.2-m telescopes with a common focus. Three of them are designed for detection of short ultraviolet Cherenkov radiation initiated 
by cosmic radiation, gamma-rays as well as charged particles, and have solar blind photomultipliers in their focal planes. The other 3 telescopes image the flashes with 37 photomultipliers (imaging camera).

- The $1.24 \mathrm{~m}$ Ritchey-Cretien reflector (Simeiz, Crimean Astrophysical Observatory). Diameter of the second mirror is $0.35 \mathrm{~m}$ and the focal length is $14.5 \mathrm{~m}$ on an English mounting (EM-2). A synchronous driving gear is used with a quartz stabilizer for guiding.

\subsubsection{Small Telescopes.}

Small-size telescopes are listed in Table 2.

\subsection{ASTRONOMICAL PUBLICATIONS}

Ukrainian astronomers prefer to publish their work in international journals such as "Astrophysical Journal", "Astronomy and Astrophysics", "Astronomical Journal", "Solar Physics", "Icarus", etc. Indeed, such publications provide the opportunity to publicize their work to a world-wide astronomical community. A few refereed journals, which publish original papers on astronomical research are published in Ukraine:

- "Kinematics and Physics of Celestial Bodies" (in Russian and Ukrainian, since 1985, bimonthly) covers various fields of modern astronomy. The English translated version is available from Allerton Press, New York. "Space Science and Technology" (in Russian and Ukrainian, since 1995, issued quarterly) contains papers on space astronomy and physics.

The journals "Kinematics and Physics of Celestial Bodies" and "Space Science and Technolgy" are published by the publishing department of the Main Astronomical Observatory of the NASU (www.mao.kiev.ua/eng/papers_e.html).

- "Radio Physics and Radio Astronomy" (in Russian and Ukrainian, since 1995) is a quarterly journal published by the Institute of Radio Astronomy of the NASU. It covers various problems on formation, propogation and registration of radio waves in different media.

- "Bulletin of the Crimean Astrophysical Observatory" has been published by this observatory since 1947. Ninety-nine volumes have already been published. Starting with volume 57 published in 1977, the "Bulletin of the Crimean Astrophysical Observatory" is translated into English and distributed by the Allerton Press, New York.

- "Odessa Observatory Publications" (in English, 2 issues per year) contains publications of original astronomical research and papers presented at international conferences. 
TABLE 2. Small-size telescopes of Ukraine

\begin{tabular}{|c|c|}
\hline $\begin{array}{l}\text { Main Astronomical Observatory, } \\
\text { NAS of Ukraine }\end{array}$ & $\begin{array}{l}\text { AZT-2 }(80 \mathrm{~cm}) \\
\text { GPS station }(1 \mathrm{~m}) \\
\text { Axial Meridian Circle } \\
\text { Twin astrograph }(0.7 \mathrm{~m}) \\
2 \text { GPS stations }(1 \mathrm{~m})\end{array}$ \\
\hline $\begin{array}{l}\text { Crimean Astrophysical Observatory, } \\
\text { MES of Ukraine }\end{array}$ & $\begin{array}{l}\operatorname{AZT}(0.5 \mathrm{~m}) \\
\operatorname{MTM}-500(0.5 \mathrm{~m}) \\
\operatorname{AZT}-8(0.7 \mathrm{~m})\end{array}$ \\
\hline $\begin{array}{l}\text { Astronomical Observatory, } \\
\text { Shevchenko National } \\
\text { University of Kyiv }\end{array}$ & $\begin{array}{l}\text { AZT-8 }(0.7 \mathrm{~m}), \text { AZT-14 }(0.5 \mathrm{~m}) \\
\text { Twin astrograph }(0.4 \mathrm{~m}) \\
\text { Horizontal Solar Telescope }(0.8 \mathrm{~m})\end{array}$ \\
\hline $\begin{array}{l}\text { Astronomical Observatory, } \\
\text { I.I.Mechnikov National } \\
\text { University of Odesa }\end{array}$ & $\begin{array}{l}1 \mathrm{~m} \text { and } 0.6 \mathrm{~m} \text { telescopes } \\
\text { two } 0.8 \mathrm{~m} \text { telescopes } \\
\text { two } 0.5 \mathrm{~m} \text { telescopes }\end{array}$ \\
\hline $\begin{array}{l}\text { Institute of Astronomy, } \\
\text { V.N.Karazin National } \\
\text { University of Kharkiv }\end{array}$ & AZT-8 $(0.7 \mathrm{~m})$ \\
\hline $\begin{array}{l}\text { Mykolaiv Astronomical Observatory, } \\
\text { MES of Ukraine }\end{array}$ & $\begin{array}{l}\text { Axial Meridian Circle } \\
\text { Multi-Channel Telescope } \\
\text { GPS station }(1 \mathrm{~m})\end{array}$ \\
\hline $\begin{array}{l}\text { Astronomical Observatory, } \\
\text { I.Franko National } \\
\text { University of L'viv }\end{array}$ & AZT-2 $(0.8 \mathrm{~m})$ \\
\hline $\begin{array}{l}\text { Space Research Laboratory } \\
\text { Uzhgorod National University }\end{array}$ & SPL-telescope $(1 \mathrm{~m})$ \\
\hline $\begin{array}{l}\text { Narodna Observatory } \\
\text { Andryushivka, Zhytomir region }\end{array}$ & 60-cm ZEISS telescope \\
\hline
\end{tabular}

- The annual "Bulletin of the Shevchenko National University of Kyiv. Astronomy" contains results of research conducted by astronomers from the University observatory.

- The "Information Bulletin of the Ukrainian Astronomical Association" 
is published twice per year and contains information about current UAA activity as well as proceedings of UAA meetings.

Besides these scientific journals, the Main Astronomical Observatory has been publishing "The Astronomical Calendar" since 1996 ("The Short Astronomical Calendar" in 1948-1995), devoted to disseminating astronomical knowledge for amateurs and students. Another astronomical calender, "The Odessa Astronomical Calendar" is published by the Odesa Astronomical Society.

Journals such as "Our Sky" (since 1998, Kyiv Republican Planetarium), "Universe: Space and Time" (since 2003, private issue), "Pulsar" (since 1998, Association of Trade Union Organizations of Students of Kyiv), as well as the Ukrainian version of the translated "Scientific American" containing many papers for nonspecialists also circulate in the popular scientific media.

\section{Current Investments in Astronomical Research}

The budgetary investments in astronomy are made by different governmental agencies. The Government of Ukraine provides through the NASU and MESU (see section 3.3) the main funding of astronomical institutions. It covers salaries of staff scientists, engineers, and other personnel as well as overhead for maintenance work on government buildings in institutions. To a lesser degree it covers expenditures for equipment, travel, and supplies.

About $30 \%$ funding is obtained from orders from the National Space Agency of Ukraine (www.nkau.gov.ua/nsau) and from other government institutions, for example, the State Fund for Basic Research (www.dffd.gov.ua). Another $10 \%$ of total funding is from individual and collaborative international grants under research programs of NATO, INTAS, STCU, CRDF, UNESCO, USAID. Besides additional salaries for employees of such collaborative projects these grants provide funding for equipment, travel, and system network development.

Total expenditures of the largest observatories are at least 2000000 USD, smaller observatories spent about of 350000 USD per year.

\section{International Cooperation}

There are at least two forms of collaboration with foreign colleagues:

Participation in international projects in the form of individual grants or projects, as collaborators. As a rule, coordinators or prinicipal investigators

are foreign scientists. Ukrainian participants obtain some financial support for short time periods (up to three months) and travel abroad. 
Collaborative projects are conducted within the framework of international research programs. A few of current projects are listed here:

- Civilian Research and Development Foundation, CRDF, project: "Spectroscopic and Photometric Monitoring of Selected Active Galactic Nuclei Objects with Extreme Properties" (Peterson, B. M., USA and Pronik, V. I., CrAO, Ukraine).

- International Association for the Promotion of Co-operation with Scientists from the New Independent States of the Former Soviet Union, INTAS, project: "The Investigation of Young Stars with Protoplanetary Disks" (Grinin V. P., CrAO).

- CRDF project: "High-energy gamma-quanta investigation with groundbased Cherenkov telescopes" (Fomin, V.P., CrAO).

- INTAS project: VLBI - astrophysical and geodetic projects with European and USA Networks (Volvatch, A.E., CrAO).

- Science and Technology Center of Ukraine, STCU, project: "Fundamental Physics and Astrophysics on-board the International Space Station: Theoretical Basis for General Relativity Tests and Astronomical Support of the Asteroid-Hazard Observational Program" (Vavilova, I.B., UAA).

Some scientists obtain international grants for long term work at foreign observatories or astronomical institutions. Due to internal rules and to protect staff positions, the foreign tenure cannot be longer than 5 months.

Several groups participate in joint projects with their own telescopes, hardware, know-how, finances and staff. This form of international participation is the most challenging.

In recent years significant contributions to world astronomy were made by: Y. Izotov, N. Guseva, L. Pilyugin, and V. Karachentseva (extragalactic astronomy); Y. Shkuratov, D. Lupishko, and V. Rozenbush (physics of planets and solar system small bodies); L. Shulman and K. Churyumov (cometary physics); Y. Yatskiv (nutation and reference frames, member of the team awarded the Descartes Prize of the European Union in 2004); R. Kostyk, N. Shchukina, N. Stepanyan, and V. Kotov (solar physics and solar-terrestrial relationship); V. Pronik and I. Pronik (AGNs); R. Hershberg, L. Lyubimkov, V. Andrievsky, and I. Andronov (physics of variable stars); A. Konovalenko (decameter radio astronomy); N. Steshenko (astronomical instrumentation). Most of their prime results were obtained due to the tight international cooperation.

\section{Scientific Investment and Priorities}

\subsection{SCIENTIFIC PRIORITIES}

Though it is a very difficult task to compose a realistic research planning document taking into account the economic situation in Ukraine, the scien- 
tific and investment priorities for Ukrainian astronomy were specified after discussions at the UAA Meeting in 2003:

- Formation and evolution of galaxies

- Global characteristics of the Sun and Sun-like-stars

- Ground-based support of space missions

- Observational and theoretical cosmology

- Physics and kinematics of solar system bodies

- Solar-planetary interactions

Understanding astronomical phenomena requires high-quality data in all frequency ranges. The activity of Ukrainian astronomical institutions is concentrated on ground-based observations in optical, centimeter and decameter wavelengths of the electromagnetic spectrum. Space mission data over a wide range of wavelengths are also used.

It is useful to distinguish short-term and medium-term investment priorities of Ukrainian astronomy.

\subsubsection{Short-term Investment Priorities}

Astronomical facilities, which are part of international networks or are involved in conducting international programs must be upgraded, in particular:

- The RT-22 radio telescope of the Crimean Astrophysical Observatory must be equipped with the new MARK4 or MARK5 recording systems and a new hydrogen frequency standard.

- R\&D for up-grading the UTR-2 decameter telescope of the Institute of Radio Astronomy must be finished and a new type of decameter antenna has to be tested. According to the decision of the NASU this work has to be done in 3 years. In the future Ukraine would like to be involved in the Square Kilometre Array, SKA, project.

- The optical 2.6-m telescope of the Crimean Astrophysical Observatory must be equipped with the same Echelle-grating spectrometer as the 2-m telescope at the high altitude observatory at Terskol Peak and with a multicolor photometer-polarimeter.

- The optical telescopes, which are used for observations of solar system small bodies, stars, and near Earth objects, NEOs, have to be equipped with new CCD-cameras and computer controlled systems.

Investments will be also allocated for establishing ground-based networks of small-size and medium-size optical telescopes, which are or will be involved in international monitoring projects for studying gravitational microlensing; ultra-rapid variability of stellar brightness and polarization; multi-wave monitoring of red dwarf flare stars and cataclysmic variable stars; magnetic activity of the Sun and solar-like stars; and pulsating stars as single objects vs components of multiple systems. 


\subsubsection{Medium-term investment priorities}

As to the medium-term priorities, the attention will be paid to participation of Ukrainian astronomical institutions in space missions, e.g., SpectrumRadio Astron (2006), Spectrum-UV (WSO) (2008); preparation of the SolarOriented Telescope (SOT) in the framework of the International Living with a Star Program; and a prospective Lunar space mission. Some projects are envisaged for the International Heliophysical Year.

Special attention will be paid to the development of the Ukrainian Virtual Observatory as a part of the International Virtual Observatory Alliance. For example, the work concerning estabvishment of the Virtual X-ray and Gamma Observatory (VIRGO) as Ukrainian part of the INTEGRAL project has been starting in 2005 .

At the moment new large ground-based astronomical facilities are not foreseen in Ukraine.

\section{Conclusion}

Despite all problems the future of Ukrainian astronomy can be considered promising. Traditionally, Ukraine is a country with a very high level of education and culture, and basic research remains an important part of science in Ukraine. Existing observational facilities provide unique opportunities for the study of astronomical objects in a wide range of spectral regions and new generations of astronomers can use these national facilities. Ukraine is one of a few counties developing its own space technologies, launch vehicles and programs. Most of these space projects are conducted with wider international cooperation.

Globalization of science provides new opportunities for collaboration with astronomers of other countries. The main goal of Ukrainian astronomy is active participation in the development and use of large infrastructures of the international astronomical community. However, this can be achieved only if adequate support can be obtained from the government.

Astronomy as well as any field of scientific research is the innovative force of economic development. Astronomers are a unique tightly knit community of people dedicated to science, trying to understand the nature of the universe. Ukrainian astronomers are in the mainstream of world science and they have to maintain their place within it.

\section{Acknowledgements}

We thank our colleagues Shakovskaya, N. (Crimea), Stodilka, M. (Lviv), Andronov, I. (Odesa), Tsymbal, V. (Simpheropol), for their help and information. We thank Yatskiv, Y. (Kyiv) for helpful remarks and enlightening discussions. YP's studies were partially supported by PPARC, Royal So- 
ciety, Leverhulme Trust. I.V. thanks the Oxford University for hospitality during the completion of the final version of this paper.

\section{References}

Nikolaenko, S. M. (2004) Education and Science: legislative and methodological basics Politechnika, pp. 1-280.

Vavilova, I. B., Yatskiv, Y. S. (2003). Astronomy education in Ukraine: status, perspectives, and activity of the Ukrainian Astronomical Association. Teaching of Astronomy in Asian- Pacific Region Bulletin 19, pp 47-48.

Yatskiv, Y. S., Vavilova, I. B., (2003) Astronomy in Ukraine: Overview of the situation and strategic planning for 2004 - 011. Kinematics and Physics of Celestial Bodies 19, No. 6, pp 569-574.

Yatskiv, Y. S., (2004) Sci. \& Tech. sphere of Ukraine: overall performance. Scientific World 5, pp. 8-13.

http://www.cia.gov/cia/publications/factbook/geos/up.html. 